\title{
Continuous Non-singular Terminal Sliding Mode Control for Magnetic Levitation Systems
}

Junxiao Wang ( $\square$ wangjunxiao19860128@126.com )

Zhejiang University of Technology https://orcid.org/0000-0002-0573-5597

\section{Qinkun Lu}

Zhejiang University of Technology Chaohui Campus: Zhejiang University of Technology

Jun Yang

Loughborough University Faculty of Engineering: Loughborough University

\section{Research Article}

Keywords: Continuous non-singular terminal sliding mode control (CNTSMC), Internal model disturbance observer (IMDO), Multiple disturbances, Disturbance rejection.

Posted Date: January 31st, 2022

DOI: https://doi.org/10.21203/rs.3.rs-1186551/v1

License: @ (i) This work is licensed under a Creative Commons Attribution 4.0 International License.

Read Full License 


\title{
Internal Model Disturbance Observer Based Continuous Non-singular Terminal Sliding Mode Control for Magnetic Levitation Systems
}

\author{
Junxiao Wang · Qinkun Lu · Jun Yang
}

Received: date / Accepted: date

\begin{abstract}
For attenuating the chattering phenomenon and improving the disturbance suppression for the magnetic levitation control system subject to multiple disturbances and parameter uncertainties, this paper proposes an internal model disturbance observer (IMDO) based continuous non-singular terminal sliding mode control (CNTSMC) scheme. Firstly, a nonlinear dynamic model of the magnetic levitation system is constructed. Then, for ensuring the system position state tracking performance and suppress the chattering phenomenon, a CNTSMC method is designed to make the system state converge to the desired position in finite time. For improving the multiple disturbance rejection, an IMDO based CNTSMC scheme is designed for the system affected by multiple disturbance which includes both high-order and trigonometric function forms. Compared with CNTSM$\mathrm{C}$ and CNTSMC+GPIO controller, simulation and experimental results show that the proposed method could improve the position tracking performance and obtains better multiple disturbance rejection. Some control performance indexes for quantitative comparison are also given to supports these results.
\end{abstract}

Keywords Continuous non-singular terminal sliding mode control (CNTSMC) - Internal model disturbance observer (IMDO) · Multiple disturbances · Disturbance rejection.

\section{J Wang}

the College of Information Engineering, Zhejiang University of Technology, Hangzhou 310023, China

E-mail: wangjunxiao19860128@126.com

Q Lu

the College of Information Engineering, Zhejiang University of Technology, Hangzhou 310023, China

E-mail: lqk19818502967@163.com

J Yang

the Department of Aeronautical and Automotive Engineering, Loughborough University, Loughborough LE11 3TU, UK

E-mail: J.Yang3@lboro.ac.uk

\section{Introduction}

Owing to the advantages of no mechanical abrasion, no pollution, and no lubrication for magnetic levitation technique, it has been applied in many industrial systems, such as magnetic levitation trains [1], magnetic levitation bearings [2], magnetic levitation wind turbines [3] and so on. However, the magnetic levitation system model is also strong nonlinear, it exists parameter uncertainties and is easy to be affected by external forces or disturbances, all of them have brought great challenges to the high-performance requirement of magnetic levitation system. In order to obtain satisfactory performance, many control methods have been proposed, such as PID control [4],[5], model predictive control [6], robust control [7],[8], feedback linearization control [9],[10], active disturbance rejection control [11], neural network control [12], fractional order control [13]-[15] and so on [16],[17]. These methods could improve the system control performance from different aspects.

Due to the strong robustness to disturbances and uncertainties, sliding mode control has been widely used in the control field during recent years [18]-[21]. However, two problems always exist in traditional sliding mode control: 1) chattering phenomenon; 2) infinite convergence time. Therefore, the continuous sliding mode control methods are proposed to suppress chattering by means of replacing the discontinuous control law with a continuous one [22]-[28]. Meanwhile, the terminal sliding mode control (TSMC) used to achieve finite time convergence has been developed [23], and adaptive fast terminal sliding mode control is applied to the magnetic levitation system successfully in [24]. Because terminal sliding mode control have the singularity problem, which makes the control input infinity, the non-singular terminal sliding mode control (NTSMC) has been proposed and used in [25]-[26]. Considering the existed chattering characteristics, a continuous non-singular terminal sliding 
mode control (CNTSMC) method is constructed in [27], it not only suppresses the chattering phenomenon but also makes the system state converge in finite time.

Although the continuous sliding mode control could reduce the chattering phenomenon and make the control input to be continuous, but the anti-disturbance ability is sacrificed or depended on the gain value, it will may result in large steady-state fluctuations especially when the system is affected by strong disturbances [28]. In recent years, the disturbance compensation technology is becoming a promise, it uses observers to estimate the system uncertainties and external disturbances and then compensates it in feed-forward channel [29]. The key point is to design a suitable observers, there are many different types of disturbance observers, such as disturbance observer (DOB), equivalent-input-disturbance (EID), extended state observer (ESO), generalized proportional integral observer (GPIO) and so on[30]-[34]. It should be noticed that the above observes could only estimate one kind of disturbance from theoretical view, because only one kind of disturbance model information are considered during the design, but there are usually many different kinds of disturbance for practical engineering. In this paper, a more accurately multiple disturbance estimation based on internal mode disturbance observer (IMDO) is proposed, it can estimate multiple disturbances which include the form of highorder and trigonometric functions.

Above all, for improving multiple disturbance rejection without sacrificing steady state performance, an IMDO based continuous terminal sliding mode controller is proposed for magnetic leviation system, the proposed control scheme could not only obtain better multiple disturbance suppression performance, but also ensure the steady state performance with continuous control input. The main contributions could be summarized as three folds:

- Firstly, a continuous non-singular terminal sliding mode controller is constructed to suppresses the chattering phenomenon, and the system state can converge to set position in finite time.

- Secondly, a novel internal model disturbance observer is designed for estimating the various forms of disturbances for the magnetic levitation system. When high-order and trigonometric function disturbances are estimated, it could help feed-forward compensation based controller obtain higher tracking accuracy and better disturbance rejection abilities.

- Thirdly, compared with existed control scheme, the simulation and experimental results are verified that not only the disturbance rejection is improved, but also steady state performance is also ensured by this method.

The organization of this paper is as follows. Section II introduces the building of nonlinear system model. Section III gives the proposed controller design and stability analysis. Section IV and V are the simulation and experimental

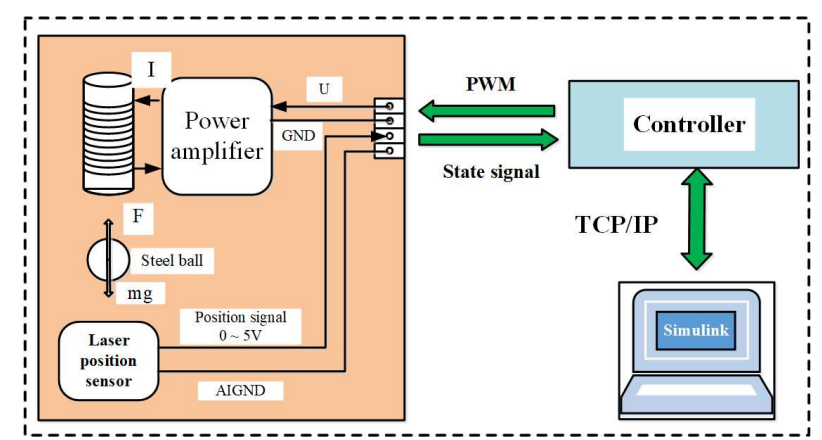

Fig. 1 Magnetic levitation control system structure.

results and corresponding quantitative analysis. Finally the conclusion end this research.

\section{Magnetic levitation system model}

In this paper, the magnetic levitation ball control system structure is shown as Fig. 1. The way it works is to change the electromagnetic force by adjusting the current in the coil, so as to stabilize the ball at a fixed position or drive the ball to track the reference trajectory. the model of magnetic levitation ball system can be written as the following explicit form

$\left\{\begin{array}{l}m \frac{d^{2} x(t)}{d t^{2}}=F\left(i_{c}, x\right)+m g \\ F\left(i_{c}, x\right)=K\left(\frac{i_{c}}{x}\right)^{2}, K=-\frac{\mu_{0} A N^{2}}{4} \\ u(t)=R i+L \frac{d i_{c}}{d t} \\ m g+F\left(i_{c 0}, x_{0}\right)=0\end{array}\right.$

where $x$ denotes instantaneous gap between electromagnet surface and the steel ball, $i_{c}$ denotes instantaneous, $F\left(i_{c}, x\right)$ denotes the electromagnetic force, $u(t)$ denotes the input voltage of the power amplifier, $g$ denotes the acceleration due to gravity, $L$ denotes the self-inductance of electromagnetic coil and $F\left(i_{c 0}, x_{0}\right)$ denotes the electromagnetic force at the equilibrium point. The rest of the parameters are referred to Table 1.

The third equation in Eq.(1) describes that the relationship among $F\left(i_{c}, x\right)$ and $i_{c}, x$ is nonlinear, $F\left(i_{c}, x\right)$ is expanded at the equilibrium point $\left(i_{c 0}, x_{0}\right)$ for the Taylor series, i.e.

$$
\begin{aligned}
F\left(i_{c}, x\right)= & F\left(i_{c 0}, x_{0}\right)+F_{i}\left(i_{c 0}, x_{0}\right)\left(i_{c}-i_{c 0}\right)+ \\
& F_{x}\left(i_{c 0}, x_{0}\right)\left(x-x_{0}\right)+F_{h}\left(i_{c}, x\right)
\end{aligned}
$$

where $F_{i_{c}}=\left.\frac{\partial F}{\partial i_{c}}\right|_{\left(i_{c 0}, x_{0}\right)}=K \frac{2 i_{c 0}}{x_{0}{ }^{2}}, F_{i_{c}}=\left.\frac{\partial F}{\partial x}\right|_{\left(i_{c 0}, x_{0}\right)}=-2 K \frac{i_{c 0}^{2}}{x_{0}{ }^{3}}$, $F_{h}\left(i_{c}, x\right)$ is the high-order term of $F\left(i_{c}, x\right)$. Then system (1) can be rewritten as

$m \ddot{x}=F_{i_{c}}\left(i_{c 0}, x_{0}\right)\left(i_{c}-i_{c 0}\right)+F_{x}\left(i_{c 0}, x_{0}\right)\left(x-x_{0}\right)$

$+F_{h}\left(i_{c}, x\right)$ 
Considering voltage signal as control input signal and bounded external disturbance signal $d(t)$, we can obtain

$\ddot{x}=g\left(i_{c}, x, d, t\right)+a_{0} x+b_{0} u$

where $a_{0}=-\frac{2 K i_{c 0}{ }^{2}}{m x_{0}{ }^{3}}, b_{0}=\frac{2 K i_{c 0}}{m x_{0}{ }^{2} R}, g\left(i_{c}, x, d, t\right)=-\frac{2 K i_{c 0} L}{m x_{0}{ }^{2} R} \frac{d i_{c}}{d t}+$ $\frac{1}{m} F_{h}\left(i_{c}, x\right)+d(t)$. Let $x_{1}=x, \dot{x}_{1}=x_{2}, y=x_{1}+n$, so the state space equation of the system can be written as

$$
\left\{\begin{array}{l}
\dot{x}_{1}=x_{2} \\
\dot{x}_{2}=a x_{1}+b u+f\left(i_{c}, x_{1}, u, d, t\right) \\
y=x_{1}+n
\end{array}\right.
$$

where $n$ is the measurement noise, $y$ is the measurement output, $f\left(i_{c}, x_{1}, u, d, t\right)=g\left(i_{c}, x_{1}, d, t\right)+\left(a_{0}-a\right) x_{1}+\left(b_{0}-b\right) u$ is the lumped disturbance which composed of unknown internal dynamics and external disturbance, $a, b$ are approximate values of $a_{0}, b_{0}$ and tunable.

Because the magnetic levitation ball system is easy to be affected by unknown disturbances and its parameters are time-varying, it is difficult to obtain accurate model of the system and achieve accurate control. In following section$\mathrm{s}$, an IMDO based continuous non-singular terminal sliding mode controller has been proposed and the stability of closeloop system has been confirmed.

\section{Controller Design}

In this section, a continuous non-singular terminal sliding mode control scheme is designed for the magnetic levitation system (5).

3.1 Internal mode disturbance observer design for magnetic levitation system

Firstly, the following definitions and lemma for concision is presented.

Define

$A \triangleq\left[\begin{array}{cc}0_{(n-1) \times 1} & I_{(n-1) \times(n-1)} \\ -1_{1 \times 1} & \alpha_{1 \times(n-1)}\end{array}\right] \in \mathfrak{R}_{n \times n}$

where

$\alpha \stackrel{\Delta}{=}-\left[C_{n}^{(n-1)} C_{n}^{(n-2)} \ldots C_{n}^{1}\right] \in \Re_{1 \times(n-1)}$

noting that $A$ is Hurwitz matrix with -1 as its multiple eigenvalues, there exists a positive definite matrix

$P \in \Re_{n \times(n)}$

such that

$A^{T} P+P^{T} A=-I$
Define $\lambda_{\max }(P)$ and $\lambda_{\min }(P)$ as the maximum and minimum real part of its eigenvalues, respectively. Define $L$ as the Laplace transformation operator and $L^{-1}$ as its inverse operator.

\section{Lemma 1 [34] For system}

$Y(s)=U(s) /(s+\lambda)^{n}\left(\lambda>\lambda_{\max }(P)\right), n \in \mathbb{N}_{+}$

where $Y(s) \triangleq$ such that $|u(t)| \leq \theta$, then

$\lim _{t \rightarrow \infty}\left|y^{(i)}(t)\right| \leq \frac{\lambda_{\max }(P)}{\sqrt{\lambda_{\min }(P)}} \theta \frac{\lambda^{-n+1+i}}{\sqrt{\lambda-\lambda_{\max }(P)}}(i=0,1, \ldots, n-1)$.

Assumption 1 There exist positive constants $\theta_{i}$ and $N \in \mathbb{N}$, such that $\left|x_{p}{ }^{(i)}\right| \leq \theta_{i}, \forall i, p \in \mathbb{N}, i \geq N$.

Secondly, extend the system state of (5) as Eq. (6) and construct GPIO as Eq. (7),

$\left\{\begin{array}{l}\dot{x}_{1}=x_{2} \\ \dot{x}_{2}=a x_{1}+b u+x_{3} \\ \dot{x}_{3}=x_{4} \\ \dot{x}_{4}=h(t)\end{array}\right.$

where $x_{3}=f\left(i_{c}, x_{1}, u, d, t\right)$ as total disturbance, $h(t)$ is a function. From Assumption 1, $x_{3}, x_{4}$ are bounded. Considering the time-varying disturbances, the GPI observer was designed as follows

$\left\{\begin{array}{l}e_{1}=z_{1}-x_{1} \\ \dot{z}_{1}=-\beta_{1} e_{1}+z_{2} \\ \dot{z}_{2}=-\beta_{2} e_{1}+a x_{1}+b u+z_{3} \\ \dot{z}_{3}=-\beta_{3} e_{1}+z_{4} \\ \dot{z}_{4}=-\beta_{4} e_{1}\end{array}\right.$

where $\beta_{1}, \beta_{2}, \beta_{3}, \beta_{4}$ is the observer gain. $z_{1}, z_{2}, z_{3}, z_{4}$ are estimates for $x_{1}, x_{2}, x_{3}, x_{4}$ respectively.

Let the observer's estimated error be $e_{2}=z_{2}-x_{2}, e_{3}=$ $z_{3}-x_{3}, e_{4}=z_{4}-x_{4}$. The observer error system is as follows

$\left\{\begin{array}{l}\dot{e}_{1}=-\beta_{1} e_{1}+e_{2} \\ \dot{e}_{2}=-\beta_{2} e_{1}+e_{3} \\ \dot{e}_{3}=-\beta_{3} e_{1}+e_{4} \\ \dot{e}_{4}=-\beta_{4} e_{1}-\ddot{x}_{3}\end{array}\right.$

The above formula can be expressed as a state space model $\dot{e}=E e+\psi$

where $e=\left[e_{1}, e_{2}, e_{3}, e_{4}\right]^{T}, \psi=\left[0,0,0,-\ddot{x}_{3}\right]^{T}$,

$E=\left[\begin{array}{llll}-\beta_{1} & 1 & 0 & 0 \\ -\beta_{2} & 0 & 1 & 0 \\ -\beta_{3} & 0 & 0 & 1 \\ -\beta_{4} & 0 & 0 & 0\end{array}\right]$

The characteristic polynomial of the observer error system is $\operatorname{det}(\lambda I-E)=\lambda^{4}+\beta_{1} \lambda^{3}+\beta_{2} \lambda^{2}+\beta_{3} \lambda+\beta_{4}$, According 
Lemma 2, chose suitable $\beta_{i}, i=1,2,3,4$, then the observer error system is local ISS. Because $\ddot{x}_{3}$ is bounded from Assumption 1. Therefore, according to Routh Criterion the designed GPI observer is asymptotically stable. The observer error will converge to zero only if $\lim _{t \rightarrow 0} \ddot{x}_{3}(t)=0$, such that when $t \rightarrow \infty, e_{1}, e_{2}, e_{3}, e_{4} \rightarrow 0$.

Thirdly, for practical system, there always coexist different kinds of disturbance which may include the forms of sine, cosine, and higher-order perturbations. Considering the multiple disturbance model, define $x_{3}+x_{5}=f\left(i, x_{1}, u, d, t\right)$, where $x_{3}=A \sin (\omega t+\varphi), x_{5}=j_{1} t^{N}+j_{2} t^{N-1}+\ldots+j_{N} t+$ $j_{N+1}$, where $A, \omega, \varphi$ and $j_{p}$ are constants, and $p=1,2, \ldots, N+$ 1 , then the extended system can be expressed as follows:

$$
\left\{\begin{array}{l}
\dot{x}_{1}=x_{2}, \\
\dot{x}_{2}=a x_{1}+b u+x_{3}+x_{5}, \\
\dot{x}_{3}=x_{4}, \dot{x}_{4}=-\omega^{2} x_{3}, \\
\dot{x}_{5}=x_{6}, \ldots, \dot{x}_{i+4}=x_{5}^{(i)} .
\end{array}\right.
$$

Then, the IMDO is designed as (9)

$$
\left\{\begin{array}{l}
\dot{z}_{1}=-\beta_{1}\left(z_{1}-x_{1}\right)+z_{2}, \\
\dot{z}_{2}=-\beta_{2}\left(z_{1}-x_{1}\right)+a x_{1}+b u+z_{3}+z_{5}, \\
\dot{z}_{3}=-\beta_{3}\left(z_{1}-x_{1}\right)+z_{4}, \\
\dot{z}_{4}=-\beta_{4}\left(z_{1}-x_{1}\right)-\omega^{2} z_{3}, \\
\dot{z}_{5}=-\beta_{5}\left(z_{1}-x_{1}\right)+z_{6}, \\
\vdots \\
\dot{z}_{N+4}=-\beta_{N+4}\left(z_{1}-x_{1}\right) .
\end{array}\right.
$$

Let $e_{i}=z_{i}-x_{i}$, where $i=1,2, \ldots, N$, obtain the observe estimate system as follows

$$
\dot{e}=\phi e+\xi d
$$

i.e.

$$
\dot{e}=\left[\begin{array}{cccccccc}
-\beta_{1} & 1 & 0 & 0 & 0 & 0 & \ldots & 0 \\
-\beta_{2} & 0 & 1 & 0 & 1 & 0 & \ldots & 0 \\
-\beta_{3} & 0 & 0 & 1 & 0 & 0 & \ldots & 0 \\
-\beta_{4} & 0 & -\omega^{2} & 0 & 0 & 0 & \ldots & 0 \\
-\beta_{5} & 0 & 0 & 0 & 0 & 1 & \ldots & 0 \\
. & . & . & . & . & . & 0 & 0 \\
. & . & . & . & . & . & . & 0 \\
. & . & . & . & . & . & & 0 \\
-\beta_{N+4} & 0 & 0 & 0 & 0 & 0 & \ldots & 0
\end{array}\right] e+\xi d
$$

where $e=\left[e_{1}, e_{2}, \ldots, e_{N+4}\right]^{T}, \xi=\left[0_{1 \times(N+3)} 1_{1 \times 1}\right], d=-x_{5}^{(N)}$. Lemma 2 [30] Consider the system $\dot{\mathbf{x}}=\mathbf{F}(\mathbf{x}, \mathbf{w})=\mathbf{f}(\mathbf{x})+$ $\mathbf{p}(\mathbf{x}) \mathbf{w}$ where $\mathbf{F}: D \times D_{w} \rightarrow R^{n}$ is locally Lipschitz in $\mathbf{x}, D \in$ $R^{n}$ is a domain that contains $\mathbf{x}=0$, and $D_{w} \in R^{m}$ is a domain that contains $\mathbf{w}=\mathbf{0}$. If $\mathbf{p}(\mathbf{x})$ is continuously differentiable and the origin of system $\dot{\mathbf{x}}=\mathbf{f}(\mathbf{x})$ is asymptotically stable, then $\dot{\mathbf{x}}=\mathbf{F}(\mathbf{x}, \mathbf{w})$ is locally ISS.
By adjusting $\beta_{i}$, the eigenvalue of $\phi$ can be adjusted to the left complex half-plane. When $d(t) \equiv 0$, the observer error system (10) is exponentially stable. Therefore, the system (10) is ISS with regard to $d(t)$ [35], so every state $e_{i}$ is bounded. Let $\left|x_{4}+x_{6}\right| \leq L_{e}, \tilde{L}_{e} \geq\left|-\left(\beta_{3}+\beta_{5}\right) e_{1}+e_{4}+e_{6}\right|=$ $\left|\dot{e}_{3}+\dot{e}_{5}\right|$ where $L_{e}, \tilde{L}_{e}$ are a positive constant.

According to Assumption 1, there exists a constant $\theta \geq 0$ such that $\left|x_{5}^{(i)}\right| \leq \theta(i=0,1, \ldots, N+4)$. Define $G_{d e_{1}}(s) \stackrel{\Delta}{=}$ $E_{1}\left((s) / D(s)\right.$ where $E_{1}(s)=L\left[e_{1}(t)\right]$ and $D(s)=L[d(t)]$. It is obvious that $G_{d e_{1}}(s)=\tau M^{-1} \xi$ where $\tau \stackrel{\Delta}{=}\left[1_{1 \times 1} 0_{1 \times(N+3)}\right]$ and $M \triangleq I s-\phi=\left(m_{i j}\right)$. Since $\operatorname{det}(M)=\sum_{i=1}^{N+4} m_{1 i} M_{1 i}=$ $\left(s+\beta_{1}\right) M_{11}-M_{12}$ where $M_{i j}$ is the algebraic cofactor of $m_{i j}$.

Define the eigenvalues of $\phi$ are chosen as $-\lambda$. Noting that $M^{-1}=M^{*} / \operatorname{det}(M)$ where $M^{*}$ is the adjoint matrix of $M$ and $M^{*}=\left(h_{i j}^{*}\right), m_{i j}^{*}=M_{j i}$, we have $G_{d e_{1}}(s)=m_{1(N+4)}^{*} /(s+$ $\lambda)^{(N+4)}=M_{(N+4) 1} /(s+\lambda)^{(N+4)}$.

Since $M_{(N+4) 1}$ is independent of $\beta_{i}(i=2,3, \ldots, N+4)$ and $L^{-1}\left[M_{(N+4) 1} D(s)\right]$ is also bounded from Assumption 1, then according to Lemma $1, \lim _{t \rightarrow \infty}\left|e_{1}{ }^{(i)}\right| \leq k \theta \frac{\lambda^{-N-3+i}}{\sqrt{\lambda-\lambda_{\max }(P)}}(\lambda>$ $\left.\lambda_{\text {max }}(P)\right),(i=0,1, \ldots, N+3)$ where $k$ is an independent positive constant. Define $e_{d}(t) \triangleq e_{3}(t)+e_{5}(t)$.

Noting that $e_{1}^{(2)}=-\beta_{1} e_{1}^{(1)}+e_{2}^{(1)}=-\beta_{1} e_{1}^{(1)}-\beta_{2} e_{1}+e_{d}$, then $\lim _{t \rightarrow \infty}\left|e_{d}\right| \leq \lim _{t \rightarrow \infty}\left(\left|e_{1}^{(2)}\right|+\beta_{1}\left|e_{1}^{(1)}\right|+\beta_{2}\left|e_{1}\right|\right) \leq \tilde{k} \theta \frac{\lambda^{-N-1}}{\sqrt{\lambda-\lambda_{\max }(P)}}$ where $\tilde{k}$ is also an independent positive constant. Therefore, the ultimate bound for $e_{1}$ and $e_{d}$ can be made arbitrarily small by magnifying $\lambda$.

Remark 1 From the above theory analysis, generalized proportional integral observer (GPIO) could estimate the time varying disturbance which implies the $\lim _{t \rightarrow 0} \ddot{x}_{3}(t)=0$, the extended state observer (ESO) and disturbance observer (DOB) could deal with the constant or slow varying disturbance with $\lim _{t \rightarrow 0} \dot{x}_{3}(t)=0$. For both the ESO and GPIO, the relevant disturbance model are included in the observer design and analysis. It means that in order to estimate the different kind$s$ of disturbance accurately, the relevant disturbance model information should be built. Considering more generalized form with multiple disturbances issue in this work, an internal mode disturbance observer (IMDO) is proposed, more disturbance modeling are considered in the observer design, this is the motivation for the proposed observer design.

3.2 IMDO based continuous non-singular terminal sliding mode controller design

Lemma 3 [36] Consider the following sliding surface for system(14)

$s=x_{1}{ }^{(n)}+c_{n}\left|x_{n}\right|^{\alpha_{n}} \operatorname{sgn}\left(x_{n}\right)+\ldots+c_{1}\left|x_{1}\right|^{\alpha_{1}} \operatorname{sgn}\left(x_{1}\right)$

$=\dot{x}_{n}+c_{n}\left|x_{n}\right|^{\alpha_{n}} \operatorname{sgn}\left(x_{n}\right)+\ldots+c_{1}\left|x_{1}\right|^{\alpha_{1}} \operatorname{sgn}\left(x_{1}\right)$ 


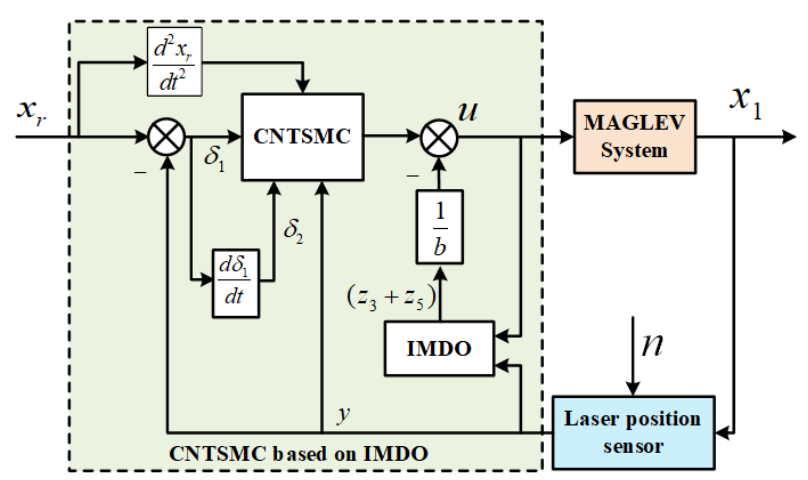

Fig. 2 Continuous non-singular sliding mode control structure based on IMDO.

where $\alpha_{i}$ and $c_{i}$ are some constants that are chosen so that the polynomial $p^{n}+c_{n} p^{n-1}+\ldots+c_{2} p+c_{1}$ is Hurwitz, i.e. all of the root are in the left-half plane. Also $\alpha_{i}$ are determined by following equations

$$
\left\{\begin{array}{l}
\alpha_{1}=\alpha_{n}=1 \\
\alpha_{i-1}=\frac{\alpha_{i} \alpha_{i+1}}{2 \alpha_{i+1}-\alpha_{i}}, i=2, \ldots, n \quad \forall n \geq 2
\end{array}\right.
$$

where $\alpha_{n+1}=1, \alpha_{n}=\alpha, \alpha \in(1-\varepsilon, 1), \varepsilon \in(0,1)$. If $u$ is determined in such a way that the system (14) reaches the sliding surface, i.e. $s=0$, then the dynamic of the system (14) becomes

$$
\left\{\begin{array}{l}
x_{1}=x_{2} \\
\dot{x}_{2}=x_{3} \\
\quad \vdots \\
\dot{x}_{n-1}=x_{n} \\
\dot{x}_{n}=c_{n}\left|x_{n}\right|^{\alpha_{n}} \operatorname{sgn}\left(x_{n}\right)+\ldots+c_{1}\left|x_{1}\right|^{\alpha_{1}} \operatorname{sgn}\left(x_{1}\right)
\end{array}\right.
$$

and the state of the system (15) converge to the zero equilibrium point $\mathbf{x}=\mathbf{0}$ from any arbitrary initial point $\mathbf{x}(\mathbf{0}) \neq \mathbf{0}$ in finite time.

Let $\delta_{1}=x_{r}-x_{1}, \delta_{2}=\dot{x}_{r}-x_{2}, x_{r}$ is the desired output. Where $\delta_{1}$ represents the tracking error of the ball position, construct the following error system according to the system (5)

$$
\left\{\begin{array}{l}
\delta_{1}=x_{r}-x_{1} \\
\dot{\delta}_{1}=\delta_{2}=\dot{x}_{r}-x_{2} \\
\dot{\delta}_{2}=\ddot{x}_{r}-\dot{x}_{2}=\ddot{x}_{r}-a x_{1}-b u-\left(x_{3}+x_{5}\right)
\end{array}\right.
$$

The sliding mode function can be designed as

$s=c_{1}\left|\delta_{1}\right|^{\alpha_{1}} \operatorname{sgn}\left(\delta_{1}\right)+c_{2}\left|\delta_{2}\right|^{\alpha_{2}} \operatorname{sgn}\left(\delta_{2}\right)+\dot{\delta}_{2}$

Theorem 1 Consider system (5) and the sliding surface in Eq. (15). The states of error system (14) will converge to the origin in finite time if the CNTSMC control law is designed as

$u=-b^{-1}\left(u_{e q}+u_{n}\right)$
Assume system (5) satisfies Assumption 1. Under the control law (16), the position tracking error of the system will converge to zero in finite time if the gain satisfies $k>L_{e}$. where

$u_{e q}=a x_{1}-\ddot{x}_{r}-c_{1}\left|\delta_{1}\right|^{\alpha_{1}} \operatorname{sgn}\left(\delta_{1}\right)-c_{2}\left|\delta_{2}\right|^{\alpha_{2}} \operatorname{sgn}\left(\delta_{2}\right)$

and $\dot{u}_{n}+T u_{n}=-\left(k+T\left|u_{n}\right|\right) \operatorname{sgn}(s), u_{n}(0)=0$, where $k, T$ are positive constants.

Proof Substituting the CNTSMC controller, the sliding mode surface could be obtained as

$s=u_{n}+x_{3}+x_{5}$

Taking the derivative of $s$, it yields

$\dot{s}=\dot{u}_{n}+\dot{x}_{3}+\dot{x}_{5}=-T u_{n}-\left(k+T\left|u_{n}\right|\right) \operatorname{sgn}(s)+\dot{x}_{3}+\dot{x}_{5}$

Taking the derivative of lyapunov function $V=\frac{1}{2} s^{2}$, it yields

$\dot{V}=s \dot{s}=s\left[-T u_{n}-\left(k+T\left|u_{n}\right|\right) \operatorname{sgn}(s)+\dot{x}_{3}+\dot{x}_{5}\right]$

$=-T u_{n} s-T\left|u_{n}\right||s|-k|s|+\left(x_{4}+x_{6}\right) s$

$\leq\left|T u_{n} s\right|-T\left|u_{n}\right||s|-k|s|+\left(x_{4}+x_{6}\right) s$

$\leq-k|s|+\left|\left(x_{4}+x_{6}\right) s\right|$

$\leq-k|s|+\left|L_{e} s\right|$

$\leq-\left(k-L_{e}\right)|s|$

where $L_{e} \geq\left|x_{4}+x_{6}\right|$. Thus, the system states could converge to origin in finite time along the sliding mode surface.

Theorem 2 Consider system (5) and the sliding surface in Eq. (15). The states of error system (14) will converge to the origin in finite time if the proposed CNTSMC+IMDO control law is designed as

$u=-b^{-1}\left(u_{e q}+u_{n}\right)$

Assume system (5) satisfies Assumption 1. Under the control law (16), the position tracking error of the system will converge to zero in finite time if the gain satisfies $k>\tilde{L}_{e}$. where

$u_{e q}=a x_{1}-\ddot{x}_{r}-c_{1}\left|\delta_{1}\right|^{\alpha_{1}} \operatorname{sgn}\left(\delta_{1}\right)$

$-c_{2}\left|\delta_{2}\right|^{\alpha_{2}} \operatorname{sgn}\left(\delta_{2}\right)+z_{3}+z_{5}$

and $\dot{u}_{n}+T u_{n}=-\left(k+T\left|u_{n}\right|\right) \operatorname{sgn}(s), u_{n}(0)=0$, where $k, T$ are positive constants.

The continuous non-singular sliding mode control structure based on IMDO (CNTSMC+IMDO) is shown as Fig .2 .

Proof Substituting Eqs. (16), (17) into (15), the sliding surface can be written as

$s=u_{n}+e_{3}+e_{5}$

where $e_{3}$ and $e_{5}$ is estimated by Eq. (10). The first derivative of sliding surface is

$\dot{s}=\dot{u}_{n}+\dot{e}_{3}+\dot{e}_{5}$
$=-T u_{n}-\left(k+T\left|u_{n}\right|\right) \operatorname{sgn}(s)+\dot{e}_{3}+\dot{e}_{5}$ 
Choosing Lyapunov function as $V=\frac{1}{2} s^{2}$, the first derivative of $V$ is

$$
\begin{aligned}
& \dot{V}=s \dot{s}=s\left[-T u_{n}-\left(k+T\left|u_{n}\right|\right) \operatorname{sgn}(s)+\dot{e}_{3}+\dot{e}_{5}\right] \\
& =-T u_{n} s-T\left|u_{n}\right||s|-k|s|+\left(\dot{e}_{3}+\dot{e}_{5}\right) s \\
& \leq\left|T u_{n} s\right|-T\left|u_{n}\right||s|-k|s|+\left(\dot{e}_{3}+\dot{e}_{5}\right) s \\
& \leq-k|s|+\left|\left(\dot{e}_{3}+\dot{e}_{5}\right) s\right| \\
& \leq-k|s|+\left|\tilde{L}_{e} s\right| \\
& \leq-\left(k-\tilde{L}_{e}\right)|s|
\end{aligned}
$$

Because $\tilde{L}_{e}$ is a positive constant, so choose $k>\tilde{L}_{e}$, then $s$ will converge to zero in finite time. Before $s$ converge to zero, it is necessary to remain system states bounded.

First, for the case $u_{n}>\left|e_{3}+e_{5}\right|$, Eq. (18) yields: $s=u_{n}+$ $e_{3}+e_{5}>0, \dot{u}_{n}=-T u_{n}-\left(k+T\left|u_{n}\right|\right) \leq-k<0, u_{n} \dot{u}_{n}<0, u_{n}$ will become smaller. Second, for the case $u_{n}<-\left|e_{3}+e_{5}\right|$, $s=u_{n}+e_{3}+e_{5}<0, \dot{u}_{n}=-T u_{n}+\left(k+T\left|u_{n}\right|\right) \geq k>0$, $u_{n} \dot{u}_{n}<0$ is also established, so $\left|u_{n}\right| \leq\left|e_{3}+e_{5}\right|$ for all time, i.e. $u_{n}$ is bounded.

Substituting Eqs. (16), (17) into (14), the error system can be written as

$$
\left\{\begin{array}{l}
\delta_{1}=x_{r}-x_{1} \\
\dot{\delta}_{1}=\delta_{2}=\dot{x}_{r}-x_{2} \\
\dot{\delta}_{2}=-c_{1}\left|\delta_{1}\right|^{\alpha_{1}} \operatorname{sgn}\left(\delta_{1}\right)-c_{2}\left|\delta_{2}\right|^{\alpha_{2}} \operatorname{sgn}\left(\delta_{2}\right)+\left(u_{n}+e_{3}+e_{5}\right)
\end{array}\right.
$$

the error system (21) can be written as state space model

$\dot{\delta}=A_{\delta}+\Phi \bar{u}$

where $\bar{u}=u_{n}+e_{3}+e_{5}, \Phi=[0,1]^{T}$

$A_{\delta}=\left[\begin{array}{c}\delta_{2} \\ -c_{1}\left|\delta_{1}\right|^{\alpha_{1}} \operatorname{sgn}\left(\delta_{1}\right)-c_{2}\left|\delta_{2}\right|^{\alpha_{2}} \operatorname{sgn}\left(\delta_{2}\right)\end{array}\right]$

Based on Lemma 3 , the system $\dot{\delta}=A_{\delta}$ is finite-time stable so that it is asymptotically stable. Furthermore $|s| \leq\left|u_{n}\right|+$ $\left|e_{3}+e_{5}\right| \leq 2\left|e_{3}+e_{5}\right| \leq 2 L_{e}$ i.e. $s$ is bouned, the error system (16) could satisfies the conditions of Lemma 2, thus it is locally ISS. This ensures the state $\delta$ is bounded.

After $s$ converge to zero, i.e. $u_{n}+e_{3}+e_{5}=0$, the error system (14) could satisfy the conditions of Lemma 3 , the state $\delta$ will converge to zero in finite time.

\section{Numerical simulation}

In this section, numerical simulations have been performed. The hardware system parameters are referred as Table I. From Table I, we calculated $a_{0}=61, b_{0}=-0.3$. The position tracking system under the control schemes (16), (17) is simulated by Matlab R2018b. Then, for comparing the tracking performance more reasonably, the convergence time $t_{c}$, maximum position tracking error $e_{\max }$, the integral of time-multiplied absolute-value of error (ITAE) [37] and the root mean square error (RMSE) [38] are used.

$$
\left\{\begin{array}{l}
I T A E=\int_{0}^{t} t\left|x_{r}-x\right| d \tau \\
R M S E=\sqrt{\int_{0}^{t} \frac{1}{t}\left(x_{r}-x\right)^{2} d \tau}
\end{array}\right.
$$

where $x_{r}$ is the set position. The calculation formula for the control performance improvement percentage as follows

Improment $=\frac{P_{C N T S M C+G P I O}-P_{C N T S M C+I M D O}}{P_{C N T S M C+G P I O}} \times 100 \%$

where $P_{C N T S M C+G P I O}$ and $P_{C N T S M C+I M D O}$ represents the performance index values of methods CNTSMC+GPIO and CNTSMC+IMDO respectively.

Table 1 Magnetic levitation system physical specifications table

\begin{tabular}{lll}
\hline Descriptions & Parameters & Nominal Values \\
\hline Ball mass & $m[k g]$ & 0.17 \\
Ball diameter & $d[m]$ & 0.06 \\
Coil Resistance & $R[\Omega]$ & 13.577 \\
Vacuum permeability & $\mu_{0}[\mathrm{H} / \mathrm{m}]$ & $4 \pi \times 10^{-7}$ \\
Magnetic permeability area & $A\left[\mathrm{~m}^{2}\right]$ & $9 \pi \times 10^{-4}$ \\
Coil turn & $N[$ Turns $]$ & 1057 \\
Static inductance & $L[m H]$ & 341 \\
Equilibrium Point & $x_{0}[\mathrm{~m}]$ & 0.0425 \\
Equilibrium Point Current & $i_{0}[\mathrm{~A}]$ & 0.633 \\
\hline
\end{tabular}

Remark 2 In order to highlight the advantages of proposed method, the suitable parameters selection with many trials has been done for every controllers. There has a tradeoff for observer parameter values, large value could cause fast convergence rate, but it may lead in noise with overlarge value. Let $N=2$, such that the order of IMDO is the same as that of GPIO. Where $\omega_{0}$ is the observer bandwidth of GPIO and IMDO, $\beta_{1}=3 \omega_{0}, \beta_{1}=3 \omega_{0}^{2}, \beta_{3}=3 \omega_{0}^{3}, \beta_{4}=100000$, $\beta_{5}=100, \beta_{6}=1000$. In addition, the three types of tests are denoted $A, B$, and $C$, respectively in Table III and $V$. A denotes the sine wave tracking performance, $B$ denotes the sine disturbance performance, $C$ denotes the performance under multi-disturbance.

\subsection{Tracking performance with uncertainties}

In order to verify the effectiveness of CNTSMC+IMDO with parameter uncertainties, change $a=55, b=-0.25$ to simulate the parameters uncertainty of the system. For a fair comparison, the CNTSMC parameters of the three methods have the same value. The parameters of three methods are shown in Table II.

Fig. 3 shows the effects of sine wave position signal tracking. As shown in Fig. 3, under the CNTSMC method, 
Table 2 Parameters of CNTSMC, CNTSMC+GPIO and CNTSM$\mathrm{C}+\mathrm{IMDO}$ in simulations

\begin{tabular}{|c|c|c|c|c|c|c|c|c|c|c|}
\hline \multirow{2}{*}{ Controller } & \multicolumn{6}{|c|}{ CNTSMC parameters } & \multicolumn{4}{|c|}{ | Oberver parameters } \\
\hline & $c_{1}$ & $c_{2}$ & $k$ & $T$ & $\alpha_{1}$ & $\alpha_{2}$ & $a$ & $b$ & $\omega_{0}$ & $\omega$ \\
\hline CNTSMC & 133 & 15 & 0.12 & 0.1 & 0.6 & 0.75 & - & - & - & - \\
\hline CNTSMC+GPIO & 140 & 20 & 0.15 & 0.1 & 0.6 & 0.75 & 61 & -0.3 & 300 & - \\
\hline CNTSMC+IMDO & 120 & 18 & 0.08 & 0.1 & 0.6 & 0.75 & 61 & -0.3 & 300 & $\pi$ \\
\hline
\end{tabular}

there is a tracking error between the system position state and the set position in the initial stage, and the control effects of the other two methods are similar. Therefore, under the influence of parameter uncertainties with different initial values, the effects of the two types of observers, GPIO and IMDO, are not much different, and both have good internal disturbances estimation performance.

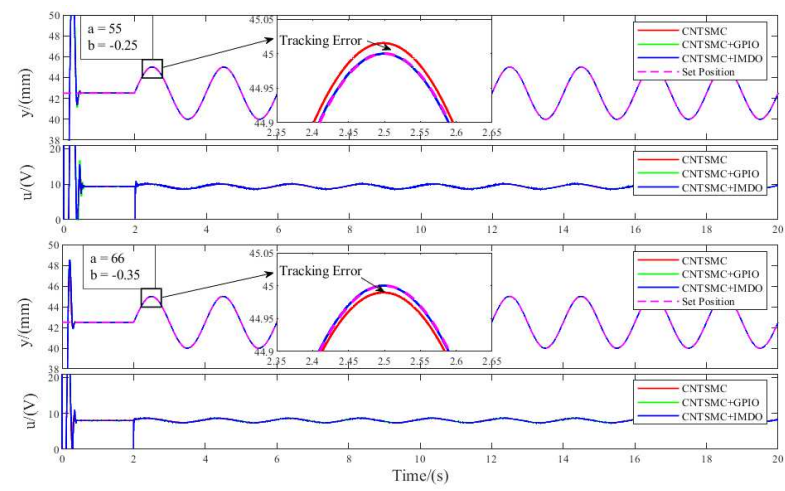

Fig. 3 Curve under sine wave tracking with parameter uncertainties (Simulation).

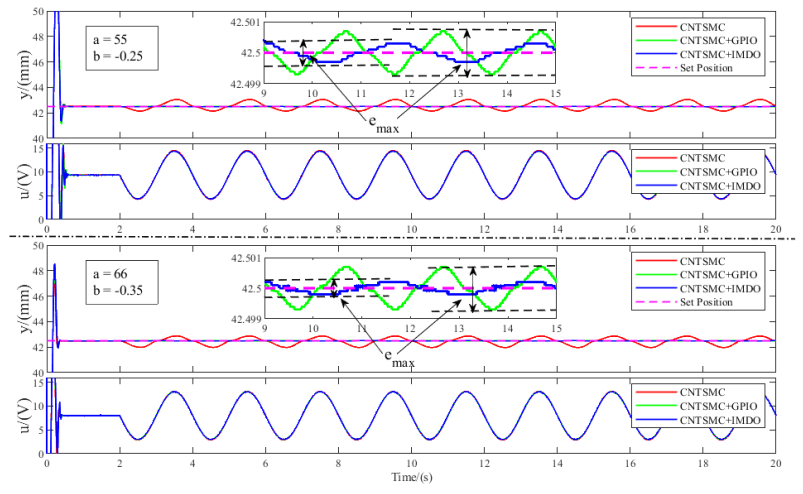

Fig. 4 Curve under sine disturbance with parameter uncertainties (Simulation).

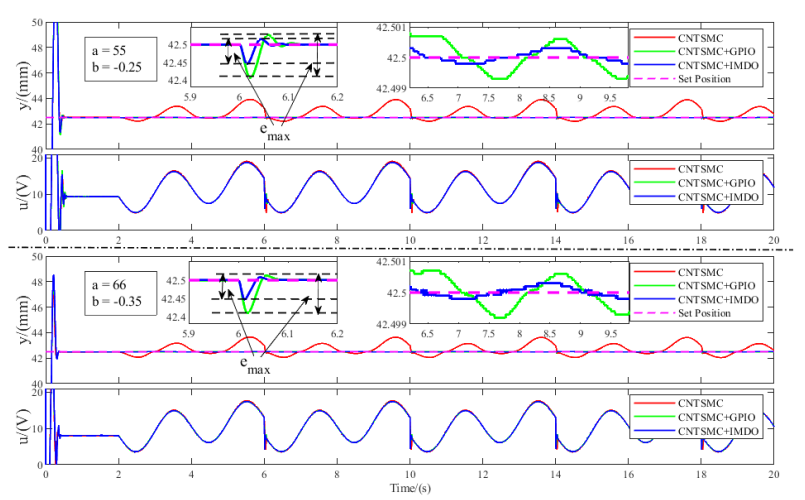

Fig. 5 Curve under multiple disturbances with parameter uncertainties (Simulation).

\subsection{Disturbance suppression performance}

In order to reflect the advantages of the method proposed in this paper, a disturbances consisting of sawtooth waves and trigonometric functions is applied at the system control input. From the response of system position states in Fig. 45 , the proposed controller inherits the strong robustness of the sliding mode controller and improves the dynamic performance and anti-interference performance of the system, especially the control performance of the system under the influence of various types of disturbances. In addition, for testing the robustness, the different parameter values of a and $b$ are set at the initial time, the same conclusion could be obtained.

Table 3 Performance of CNTSMC, CNTSMC+GPIO and CNTSM$\mathrm{C}+\mathrm{IMDO}$ in simulations

\begin{tabular}{|c|c|c|c|c|}
\hline \multirow{2}{*}{ Groups } & \multirow{2}{*}{ Controller } & \multicolumn{3}{|c|}{ Performance } \\
\hline & & ITAE & RMSE $\left(10^{-3}\right)$ & $e_{\max }(\mathrm{mm})$ \\
\hline \multirow{4}{*}{$\begin{array}{c}\text { A } \\
\text { (Fig.3) }\end{array}$} & CNTSMC & 3.1367 & 10.1999 & - \\
\hline & CNTSMC+GPIO & 1.7360 & 5.5324 & - \\
\hline & CNTSMC+IMDO & 1.7169 & 5.4518 & - \\
\hline & Improvement & $1.22 \%$ & $1.48 \%$ & - \\
\hline \multirow{4}{*}{$\begin{array}{c}\text { B } \\
\text { (Fig.4) }\end{array}$} & CNTSMC & 86.4142 & 296.0604 & 0.9029 \\
\hline & CNTSMC+GPIO & 0.1225 & 0.4189 & 0.0020 \\
\hline & CNTSMC+IMDO & 0.0608 & 0.2141 & 0.0010 \\
\hline & Improvement & $50.37 \%$ & $48.89 \%$ & $50 \%$ \\
\hline \multirow{4}{*}{$\begin{array}{c}\mathrm{C} \\
\text { (Fig.5) }\end{array}$} & CNTSMC & 152.6774 & 600.6235 & 1.7125 \\
\hline & CNTSMC+GPIO & 0.3772 & 6.2863 & 0.1202 \\
\hline & CNTSMC+IMDO & 0.1719 & 3.1697 & 0.0723 \\
\hline & Improvement & $54.43 \%$ & $49.58 \%$ & $39.85 \%$ \\
\hline
\end{tabular}

In order to highlight the advantages of this method more intuitively, the quantitative comparison as shown in Table III, the statistical bar graph is made as shown in Fig. 6-7, where $\mathrm{A}, \mathrm{B}$, and $\mathrm{C}$ represent three sets of simulation results respectively. From those figures, it is easy to find that the tracking accuracy and convergence rate of the system state are improved in presence of disturbances. 


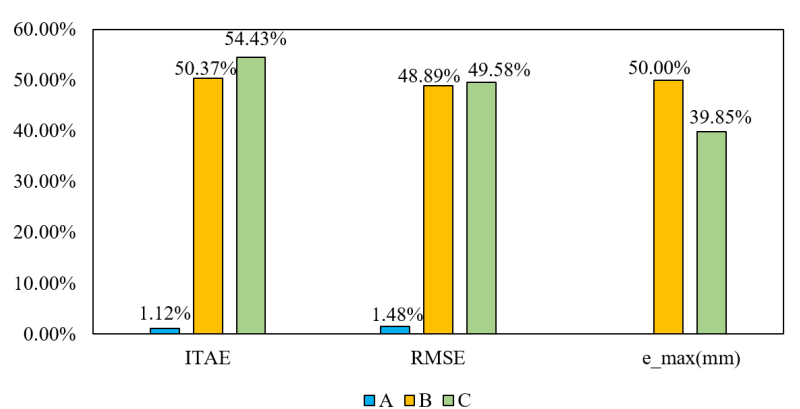

Fig. 6 Improvements of Performance in Simulations.

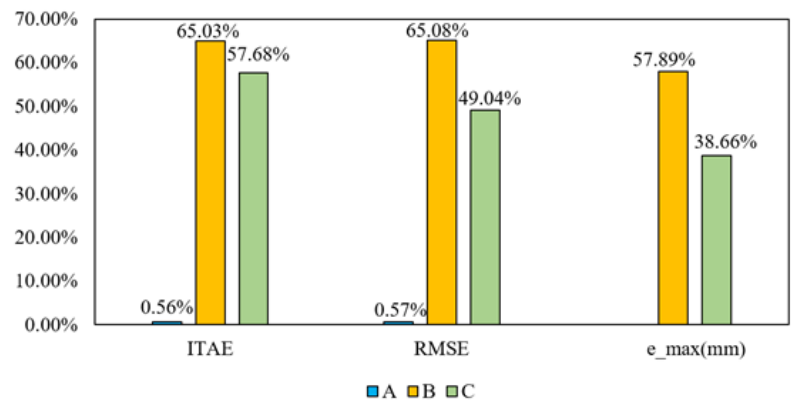

Fig. 7 Improvements of Performance in Simulations.

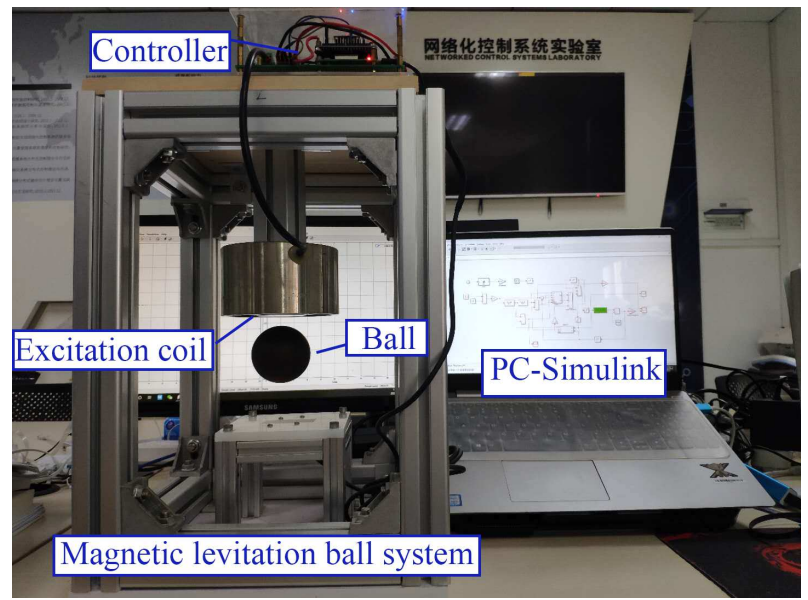

Fig. 8 Experimental test bench.

\section{Experimental results}

The test bench is shown in Fig. 8. The system hardware structure is described as Fig. 9. The uncertainties are performed by regulating the relevant parameter values which used in the controller, the disturbance is given in the control input channel. It is easy to load different disturbance such as sinusoidal wave, sawtooth wave and so on.

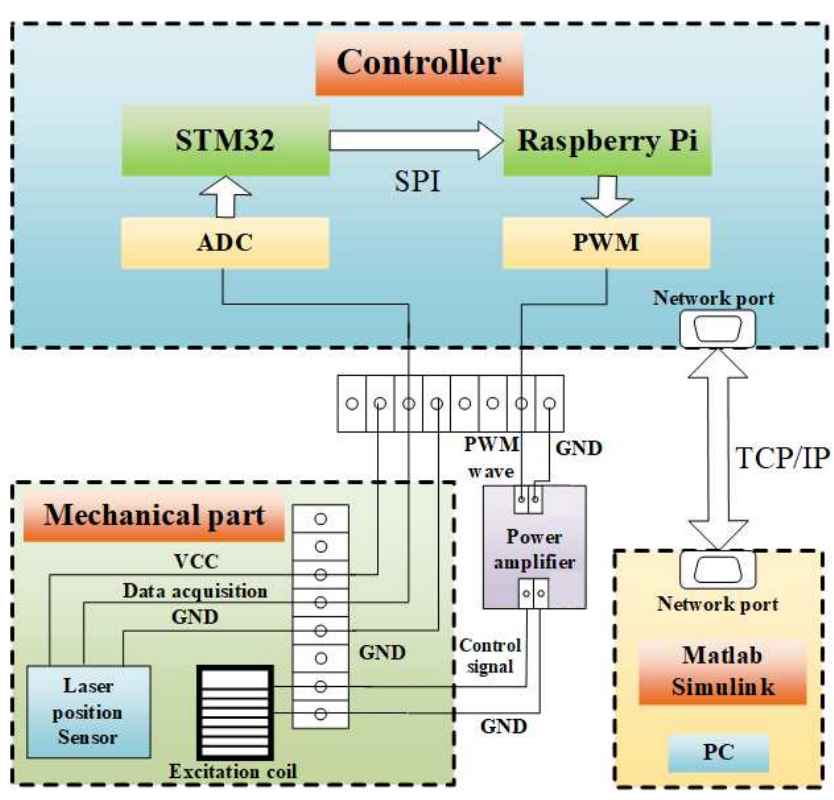

Fig. 9 Experimental system structure.

Table 4 Parameters of CNTSMC, CNTSMC+GPIO and CNTSM$\mathrm{C}+\mathrm{IMDO}$ in experiments

\begin{tabular}{|c|c|c|c|c|c|c|c|c|c|}
\hline \multirow{2}{*}{ Controller } & \multicolumn{5}{|c|}{ CNTSMC parameters } & \multicolumn{4}{|c|}{ Oberver parameters } \\
\hline & $c_{1} \quad c_{2}$ & $k$ & $T$ & $\alpha_{1}$ & $\alpha_{2}$ & $a$ & $b$ & $\omega_{0}$ & $\omega$ \\
\hline CNTSMC & 16025 & 1.2 & 0.1 & 0.6 & 0.75 & $1-$ & - & - & - \\
\hline CNTSMC+GPIO & 45030 & 0.5 & 0.1 & 0.6 & 0.75 & & -0.25 & 320 & - \\
\hline CNTSMC+IMDO & 47530 & 0.5 & 0.1 & 0.6 & 0.75 & 58 & -0.25 & 330 & $0.25 \pi$ \\
\hline
\end{tabular}
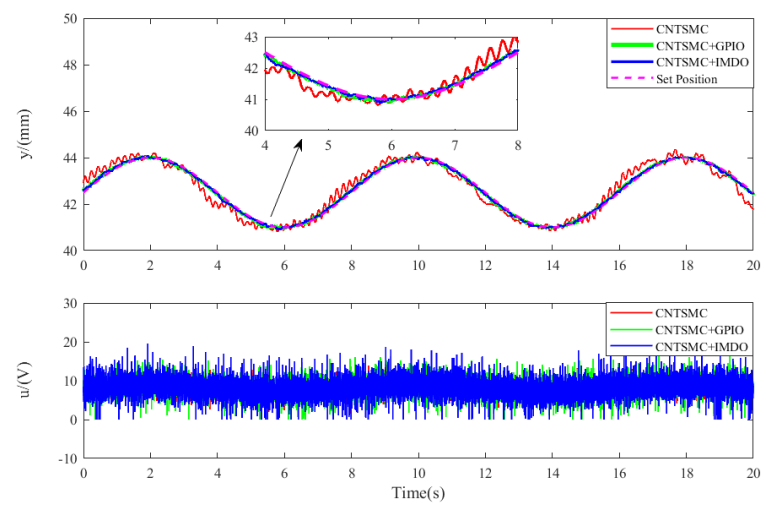

Fig. 10 Curve under sine wave tracking (Experiments).

\subsection{Tracking performance with uncertainties}

The controller parameter values are shown as Table IV. Fig. 10 shows the sine wave position tracking results, it shows that all methods can track the set position, because the fluctuation amplitude of CNTSMC curve is bigger than other two schemes. In summary, it could be concluded that CNTSMC+IMDO and CNTSMC+GPIO improve the tracking performance of the system compared to CNTSMC. 

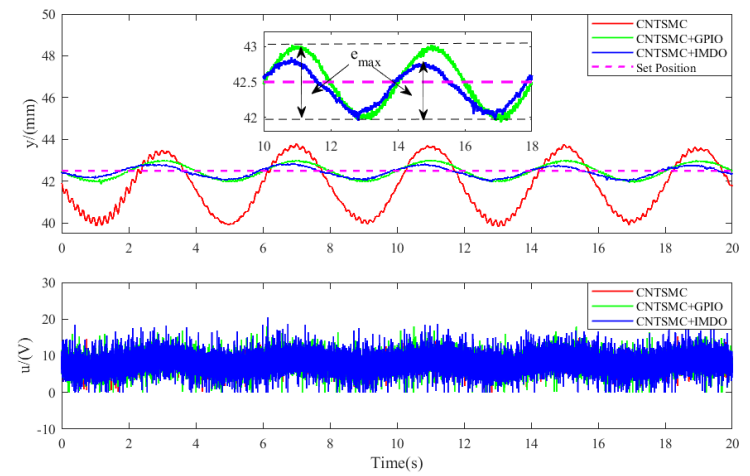

Fig. 11 Curve under sin disturbances (Experiments).
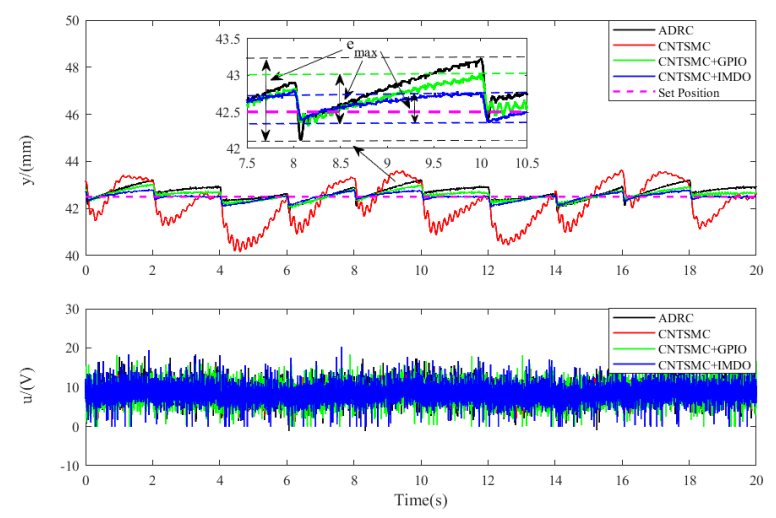

Fig. 12 Curve under multiple disturbances (Experiments).

\subsection{Disturbance suppression performance}

Figs. 11-12 shows the system response curves with sine disturbance and multiple disturbances (sine and sawtooth wave disturbances). From Fig. 11, there exists position tracking error under CNTSMC, but other two methods have better tracking performance. In addition, from the magnified figure, the convergence rate of CNTSMC+IMDO is more quickly. Fig. 12 shows the position curves under multiple disturbances, not only the convergence accuracy has been improved from Table $\mathrm{V}$, the maximum tracking error $e_{\max }$ of the proposed method is smallest $(0.4 \mathrm{~mm})$, and that of CNTSMC+GPIO and CNTSMC are $0.6 \mathrm{~mm}$ and $3.6 \mathrm{~mm}$, so the proposed method obtains better disturbance reject ability.

From above experimental results as shown in Table V, we can get the conclusion that the CNTSMC+IMDO controller has better tracking performance and obtains better anti-interference ability for the closed-loop system. From Fig. 12, notice that the multiple disturbance rejection is compared based on ESO based ADRC method, CNTSMC+GPIO, and CNTSMC+IMDO, the superiority of proposed IMDO observer is verified. Fig. 13 shows the improvement of mul-

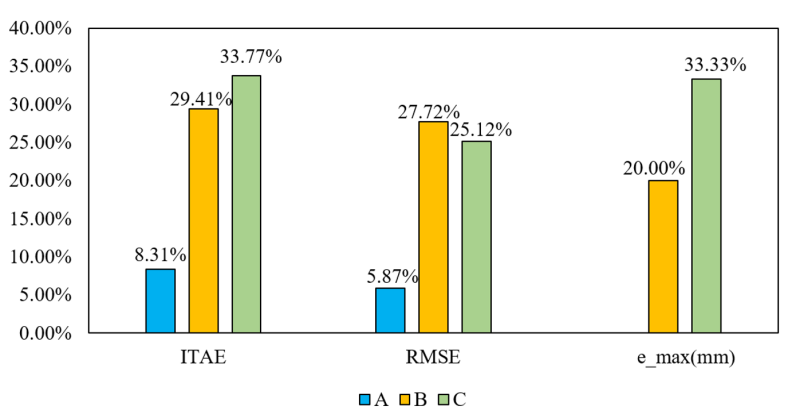

Fig. 13 Improvements of Performance in Experiments.

tiple disturbances reject performances using statistical bar graph. Due to the high frequency noise, although the experimental performance is not so good, but similar conclusions can be drawn with the comparison. Compared with CNTSMC+GPIO and CNTSMC methods, the proposed method has better tracking accuracy and convergence rate in the presence of multiple disturbances.

Table 5 Performance of CNTSMC, CNTSMC+GPIO and CNTSM$\mathrm{C}+\mathrm{IMDO}$ in experiments

\begin{tabular}{|c|c|c|c|c|}
\hline \multirow{2}{*}{ Groups } & \multirow{2}{*}{ Controller } & \multicolumn{3}{|c|}{ Performance } \\
\hline & & $I T A E$ & $R M S E$ & $e_{\max }(\mathrm{mm})$ \\
\hline \multirow{4}{*}{$\begin{array}{c}\text { A } \\
\text { (Fig.10) }\end{array}$} & CNTSMC & 93.5413 & 0.2891 & - \\
\hline & CNTSMC+GPIO & 24.3402 & 0.0695 & - \\
\hline & CNTSMC+IMDO & 22.3183 & 0.0654 & - \\
\hline & Improvement & $8.31 \%$ & $5.87 \%$ & - \\
\hline \multirow{4}{*}{$\begin{array}{c}\text { B } \\
\text { (Fig.11) }\end{array}$} & CNTSMC & 639.5383 & 1.8666 & 3.9 \\
\hline & CNTSMC+GPIO & 123.2469 & 0.3424 & 1.0 \\
\hline & CNTSMC+IMDO & 87.0026 & 0.2475 & 0.8 \\
\hline & Improvement & $29.41 \%$ & $27.72 \%$ & $20.00 \%$ \\
\hline \multirow{5}{*}{$\begin{array}{c}\mathrm{C} \\
\text { (Fig.12) }\end{array}$} & ADRC & 104.2016 & 0.3125 & 0.9 \\
\hline & CNTSMC & 303.5815 & 0.9172 & 3.6 \\
\hline & CNTSMC+GPIO & 69.0011 & 0.2063 & 0.6 \\
\hline & CNTSMC+IMDO & 45.6993 & 0.1545 & 0.4 \\
\hline & Improvement & $33.77 \%$ & $25.12 \%$ & $33.33 \%$ \\
\hline
\end{tabular}

\section{Conclusion}

The position tracking problems for magnetic levitation system with uncertainties and multiple disturbances has been focused in this paper. For implementing closed-loop control of the system, the nonlinear system mathematical model was built and the uncertainties and external disturbances was analyzed. A novel internal model disturbance observer was applied to estimate the external high-order disturbances, trigonometric function type of disturbances and parameters uncertainties. Then a composite controller has been designed by combining CNTSMC with IMDO. Compared with CNTSMC and CNTSMC+GPIO, the proposed methods has improved the steady state performance and multiple 
disturbances rejection. Some control performance indexes with quantitative comparison also supports these results.

Acknowledgements This work was supported in part by National Natural Science Foundation (NNSF) of China under Grants (61803335, 51877207).

\section{Data availability}

Data sharing is not applicable to this article as no datasets were generated or analysed during the current study.

\section{Declarations}

\section{Conflict of interest}

The authors declare that they have no conflict of interest.

\section{References}

1. H. W. Lee, K. C. Kim, J. Lee.: Review of maglev train technologies. IEEE Transactions on Magnetics, vol. 42, no. 7, pp. 19171925 (2006)

2. S. Xu, J. Fang. :A novel conical active magnetic bearing with claw structure. IEEE Transactions on Magnetics, vol. 50, no. 5, pp. 1-8 (2013)

3. G. Shrestha, H. Polinder, D. Bang, et al.:Structural flexibility: A solution for weight reduction of large direct-drive wind-turbine generators, IEEE Transactions on Energy Conversion, vol. 25, no. 3, pp: $732-740(2010)$

4. C. M. Lin, M. H. Lin, C. W. Chen.:SoPC-based adaptive PID control system design for magnetic levitation system, IEEE Systems journal, vol. 5, no. 2, pp: 278-287 (2011)

5. M. Golob, B. Tovornik.:Modeling and control of the magnetic suspension system, ISA transactions, vol. 42, no. 1, pp: 89-100 (2003)

6. T. Bachle, S. Hentzelt and K. Graichen.:Nonlinear model predictive control of a magnetic levitation system, Control Engineering Practice, vol. 21, no. 9, pp. 1250-1258 (2013)

7. Z. J. Yang, M. Tateishi.:Adaptive robust nonlinear control of a magnetic levitation system, Automatica, vol, 37, pp: 1124-1131 (2001)

8. A. Javadi, S. Pezeshki.:A new model-free adaptive controller versus non-linear $\mathrm{H} \infty$ controller for levitation of an electromagnetic system, Transactions of the Institute of Measurement and Control, vol. 35, pp. 3, pp: 321-329 (2013)

9. A. El Hajjaji, M. Ouladsine.:Modeling and nonlinear control of magnetic levitation systems, IEEE Transactions on industrial Electronics, vol. 48, no. 4, pp: 831-838 (2001)

10. Y. Qin, H. Peng, W. Ruan, et al.: A modeling and control approach to magnetic levitation system based on state-dependent ARX model, Journal of Process Control, vol. 24, no. 1, pp. 93-112 (2014)

11. W. Wei, W. Xue, D. Li.:On disturbance rejection in magnetic levitation," Control Engineering Practice, vol, 82, pp: 24-35 (2019)

12. H. An and J. Chen.:The Magnetic Levitation Ball Position Control with Fuzzy Neural Network Based on Particle Swarm Algorithm, 2018 37th Chinese Control Conference (CCC), pp. 2788-2793 (2018)

13. S. Folea, C. I. Muresan, R. De Keyser, et al.:Theoretical analysis and experimental validation of a simplified fractional order controller for a magnetic levitation system, IEEE Transactions on Control Systems Technology. vol. 24, no. 2, pp: 756-763 (2015)
14. C. I. Muresan, C. Ionescu, S. Folea, et al.:Fractional order control of unstable processes: the magnetic levitation study case, Nonlinear Dynamics, vol. 80, no. 4, pp. 1761-1772 (2015)

15. R. De Keyser, C. I. Muresan, C. M. Ionescu.:A novel auto-tuning method for fractional order PI/PD controllers, ISA transactions, vol 62, pp: 268-275 (2016)

16. T. Gluck, W. Kemmetmuller, C. Tump, et al.:A novel robust position estimator for self-sensing magnetic levitation systems based on least squares identification, Control Engineering Practice, vol. 19, no. 2, pp. 146-157 (2011)

17. R. Morales, V. Feliu, H. Sira-Ramirez.:Nonlinear control for magnetic levitation systems based on fast online algebraic identification of the input gain, IEEE Transactions on Control Systems Technology, vol. 19, no. 4, pp. 757-771 (2010)

18. Y. Shtessel, C. Edwards, L. Fridman, A. Levant.:Sliding mode control and observation. , New York: Springer New York (2014)

19. L. Yipeng, L. Jie, Z. Fengge and Z. Ming.:Fuzzy Sliding Mode Control of Magnetic Levitation System of Controllable Excitation Linear Synchronous Motor, IEEE Transactions on Industry Applications, vol. 56, no. 5, pp. 5585-5592 (2020)

20. G. Sun, L. Wu, Z. Kuang, et al.:Practical tracking control of linear motor via fractional-order sliding mode, Automatica, vol. 94, pp. 221-235 (2018)

21. R. Cui, L. Chen, C. Yang, et al.:Extended state observer-based integral sliding mode control for an underwater robot with unknown disturbances and uncertain nonlinearities, IEEE Transactions on Industrial Electronics, vol. 64, no. 8, pp: 6785-6795 (2017)

22. Y. Feng, F. Han, X. Yu.:Chattering free full order sliding mode control, Automatica, vol, 50, no. 4, pp: 1310-1314 (2014)

23. Y. Tang.:Terminal sliding mode control for rigid robots, Automatica, 1998, 34(1): 51-56.

24. N. Boonsatit, C. Pukdeboon.:Adaptive fast terminal sliding mode control of magnetic levitation system, Journal of Control, Automation and Electrical Systems, vol. 27, no. 4, pp. 359-367 (2016)

25. Y. Wu, X. Yu, Z. Man.:Terminal sliding mode control design for uncertain dynamic systems, Systems \& Control Letters, vol. 34, no. 5, pp: 281-287 (1998)

26. B. Xu, L. Zhang and W. Ji.:Improved Non-Singular Fast Terminal Sliding Mode Control With Disturbance Observer for PMSM Drives, IEEE Transactions on Transportation Electrification, vol. 7, no. 4, pp. 2753-2762 (2021)

27. H. Rabiee, M. Ataei, M. Ekramian.:Continuous nonsingular terminal sliding mode control based on adaptive sliding mode disturbance observer for uncertain nonlinear systems, Automatica, online (2019)

28. H. Wang, Y. Pan, S. Li, H. Yu.:Robust sliding mode control for robots driven by compliant actuators, IEEE Transactions on Control Systems Technology, vol. 27, no. 3, pp: 1259-1266 (2018)

29. J. Yang, S. Li, X. Yu.:Sliding-mode control for systems with mismatched uncertainties via a disturbance observer, IEEE Transactions on industrial electronics, vol. 60, no. 1, pp: 160-169 (2012)

30. S. Li, J. Yang, W. H. Chen, et al.:Disturbance observer-based control: methods and applications, CRC press (2016)

31. Q. Mei, J. She, Z. Liu, Y. Xiong, W. He and D. Li.:Disturbance Rejection Based on Equivalent-Input-Disturbance Approach Using High-Order Filter, 2021 IEEE International Conference on Mechatronics (ICM), pp. 1-5, (2021)

32. J. Wang, S. Li, J. Yang, et al.:Extended state observer-based sliding mode control for PWM-based DC-DC buck power converter systems with mismatched disturbances, IET Control Theory \& Applications, vol. 9, no. 4, pp: 579-586 (2015)

33. J. Wang, F. Wang, G. Wang, et al.:Generalized proportional integral observer based robust finite control set predictive current control for induction motor systems with time-varying disturbances, IEEE Transactions on Industrial Informatics, vol. 14, no. 9, pp: 4159-4168 (2018) 
34. Y. Yan, J. Yang, Z. Sun, et al.:Robust speed regulation for PMSM servo system with multiple sources of disturbances via an augmented disturbance observer, IEEE/ASME Transactions on Mechatronics, vol. 23, no. 2, pp: 769-780 (2018)

35. H. K. Khalil.:Nonlinear systems, Upper Saddle River (2002)

36. S. P. Bhat, D. S. Bernstein.:Geometric homogeneity with applications to finite-time stability, Mathematics of Control, Signals and Systems, vol. 17, no.2, pp. 101-127 (2005)

37. Y. Pan, T. Sun, H. Yu.:Composite adaptive dynamic surface control using online recorded data, International Journal of Robust and Nonlinear Control, vol. 26, no.18, pp. 3921-3936, (2016)

38. J. J. Rubio.:Robust feedback linearization for nonlinear processes control, ISA transactions, vol. 74, pp. 155-164 (2018) 